\title{
Systemische Therapie einer Acne conglobata mit Isotretinoin bei einem mit Lamotrigin und Valproinsäure eingestellten epileptischen Anfallsleiden
}

\author{
Systemic Treatment with Isotretinoin in a Patient with Acne Conglobata and Epilepsy Controlled with \\ Lamotrigine and Valproic Acid
}

Autoren

Institut

\section{Dume, N. Voshege, J. Freise, S. Grabbe, T. Jansen}

Klinik und Poliklinik für Dermatologie, Venerologie und Allergologie der Universität Essen (Direktor: Prof. Dr. Stephan Grabbe)

\section{Bibliografie}

DOI $10.1055 / \mathrm{s}-2007-995778$

Akt Dermatol 2008; 34 :

188-191 @ Georg Thieme

Verlag KG Stuttgart · New York ISSN 0340-2541

\section{Korrespondenzadresse}

Dr. Thomas Jansen

Klinik und Poliklinik für

Dermatologie, Venerologie

und Allergologie der

Universität Essen

Hufelandstraße 55

45122 Essen

thomas.jansen@

medizin.uni-essen.de

\section{Zusammenfassung \\ $\nabla$}

Bei einem 21-jährigen Patienten mit Acne conglobata und epileptischem Anfallsleiden, das mit Lamotrigin und Valproinsäure eingestellt war, wurde eine Systemtherapie mit Isotretinoin $40 \mathrm{mg} / \mathrm{die}$ (entsprechend 0,5 mg/kg Körpergewicht/die) unter Kontrolle der Antiepileptikaspiegel eingeleitet. Bei klinisch deutlicher Be-

\section{Einleitung}

$\nabla$

Eine Vielzahl von Medikamenten kann eine Akne induzieren, aggravieren oder unterhalten. Dazu zählen auch Antiepileptika wie Hydantoine, Trimethadion, Primidon und Phenobarbital [5]. Bislang gibt es über die zugrunde liegenden Pathomechanismen der durch Antiepileptika ausgelösten Akne keine gesicherten Erkenntnisse. Bei den Hydantoinen werden androgene Eigenschaften vermutet.

Frühere Untersuchungen ließen allerdings keinen Zusammenhang zwischen Antiepileptika und Akne erkennen. So verglichen Greenwood und Mitarbeiter [4] den Schweregrad der Akne und die Sebumexkretionsrate bei 243 Patienten, die wegen einer Epilepsie unterschiedliche Medikamente einnahmen, mit 2176 gesunden Individuen. In dieser Studie war bei den Patienten weder ein signifikant höherer Schweregrad der Akne anzutreffen noch eine signifikant höhere Sebumexkretionsrate messbar.

Aufgrund von Begleiterkrankungen ist oftmals eine systemische Therapie unumgänglich, sodass in diesen Fällen die Therapie einer gleichzeitig bestehenden Akne erschwert und eine vollständige Abheilung oftmals nicht möglich ist. Im Falle von Isotretinoin (13-cis-Retinsäure), das Aknepatienten über mehrere Monate verabreicht wird, ergibt sich potenziell ein höheres Risiko von Arzneimittelinteraktionen, wenn Patienten fundbesserung konnte die Dosis nach vier Monaten auf $30 \mathrm{mg} /$ die reduziert werden. Während der Behandlung wurden keine pathologischen Verschiebungen der Antiepileptikaspiegel beobachtet. Bei gegebener Indikation sollte Patienten mit medikamentös eingestellter Epilepsie eine Systemtherapie mit Isotretinoin unter Beachtung potenzieller Arzneimittelinteraktionen nicht vorenthalten werden.

behandelt werden, die neben der Hauterkrankung gleichzeitig auch an chronischen Erkrankungen leiden und diesbezüglich einer dauerhaften Behandlung bedürfen.

Vorgestellt wird ein Patient mit Acne conglobata und medikamentös eingestelltem epileptischen Anfallsleiden, der mit einer systemischen Isotretinointherapie erfolgreich und komplikationslos behandelt wurde. Anhand des Falles soll auf die potenziellen Interaktionen zwischen Isotretinoin und Antiepileptika eingegangen werden.

\section{Fallbericht \\ $\nabla$}

Anamnese

Ein 21-jähriger Patient stellte sich in unserer Ambulanz mit einer seit Jahren bestehenden Acne conglobata vor. Der Hautbefund hatte sich in den letzten Wochen deutlich verschlechtert. Zudem litt der Patient seit dem zweiten Lebensjahr an einem epileptischen Anfallsleiden, das medikamentös mit Lamotrigin $100 \mathrm{mg} /$ die $\left(\right.$ Lamictal $^{\circledR}$ ) und Valproinsäure $1200 \mathrm{mg} /$ die $\left(\right.$ Ergenyl $^{\circledR}$ ) eingestellt war. Das Allgemeinbefinden war nicht beeinträchtigt.

\section{Hautbefund}

Im Gesicht, am Rücken sowie an der Brust fanden sich auf seborrhoischer Haut multiple entzündliche, teilweise konfluierende, follikulär ge- 


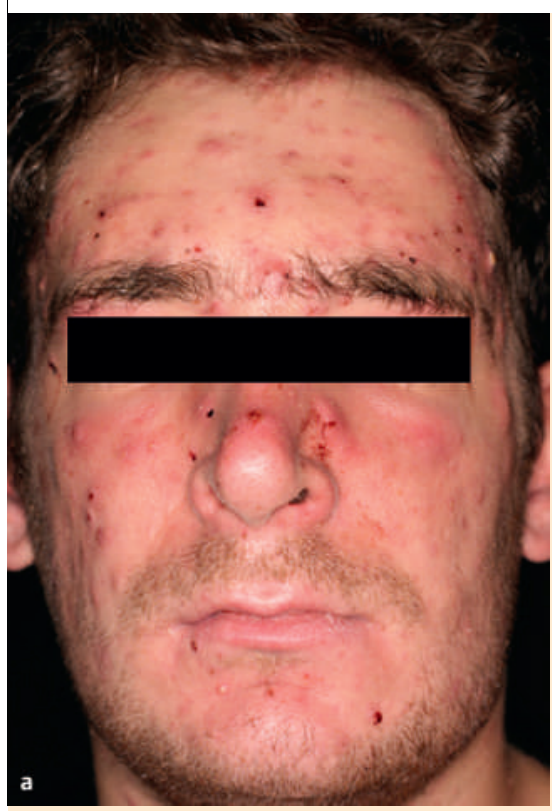

Abb. 1 21-jähriger Patient mit Acne conglobata vor (a) und nach (b) viermonatiger Systemtherapie mit Isotretinoin.

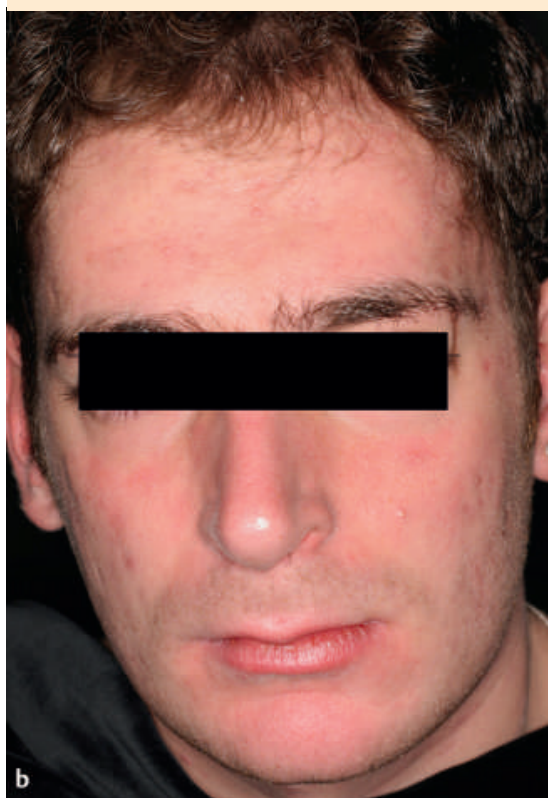

bundene Papeln, Pusteln und Knoten mit Narbenbildung ( Abb. 1 a, 2a). Vereinzelt waren Fistelkomedonen vorhanden. Dagegen waren geschlossene oder offene Komedonen nicht erkennbar.

\section{Klinisch-chemische Untersuchungen}

Bis auf leicht erhöhte Entzündungsparameter wie ein CRP-Anstieg auf $1,5 \mathrm{mg} / \mathrm{dl}$ (normal: $<0,5 \mathrm{mg} / \mathrm{dl}$ ) und eine Leukozytose von 17,02/nl (normal: 3,8-10,7/nl) unauffällige Routinelaborparameter. LH, FSH, Testosteron, DHEAS, Androstendion 17-Hydroxy-Progesteron und Kortisol im Normbereich. Lamotriginund Valproinsäurespiegel im Serum im Normbereich.

\section{Bakteriologische Untersuchung}

In mehrfachen Pustelabstrichen kultureller Nachweis von Staphylococcus epidermidis.
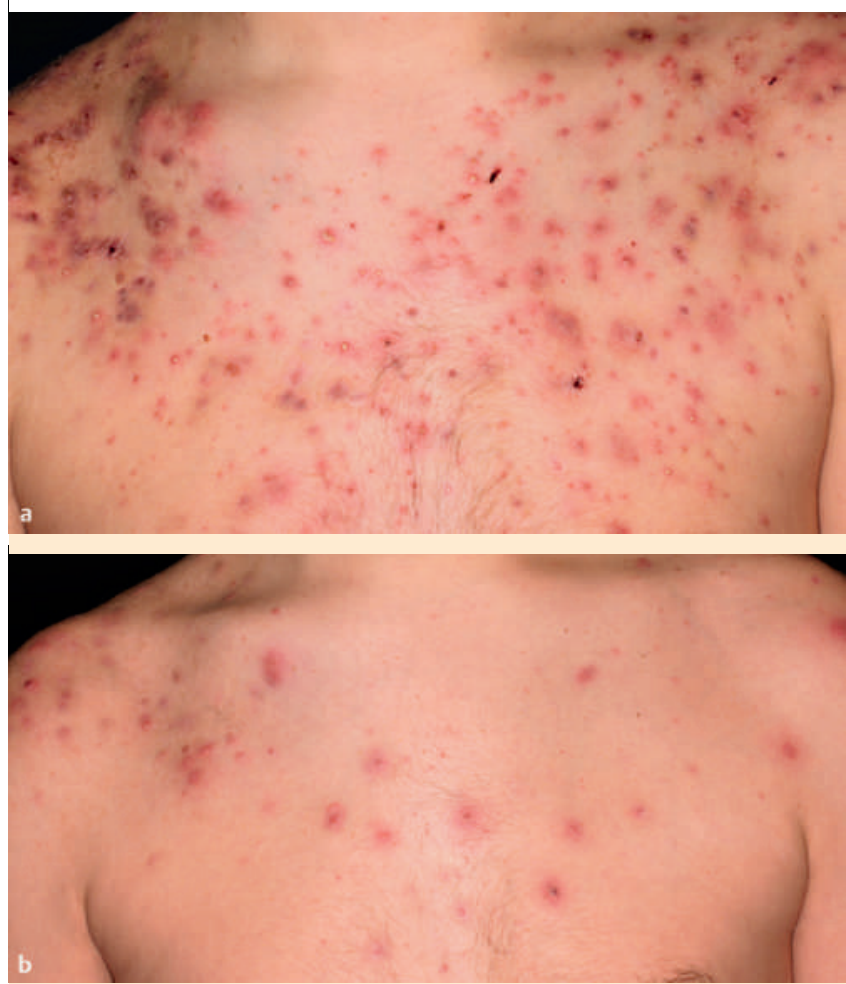

Abb. 2 21-jähriger Patient mit Acne conglobata vor (a) und nach (b) viermonatiger Systemtherapie mit Isotretinoin.

\section{Therapie und Verlauf}

$\nabla$

Aufgrund der Schwere und Ausdehnung des Hautbefundes wurde eine systemische Therapie mit $40 \mathrm{mg} /$ die Isotretinoin (entsprechend $0,5 \mathrm{mg} / \mathrm{kg}$ Körpergewicht/die) eingeleitet. Unter der angegebenen Therapie kam es zu einer deutlichen Befundbesserung mit Reduktion der entzündlichen Effloreszenzen und der Seborrhö, sodass die Therapie nach vier Monaten mit einer reduzierten Dosis von $30 \mathrm{mg} /$ die als Erhaltungsdosis fortgesetzt werden konnte ( $\mathbf{A b b} \mathbf{1} \mathbf{1}$ b,2 b). Über den gesamten Behandlungszeitraum wurden regelmäßige Verlaufskontrollen der Antiepileptikaspiegel im Serum durchgeführt, wobei keine pathologischen Veränderungen auffielen. Ein epileptisches Anfallsleiden wurde in dieser Zeit nicht beobachtet.

\section{Diskussion}

Die Einführung von Isotretinoin hat die Therapie der mittelschweren bis schweren Akneformen revolutioniert [10]. Im Unterschied zu allen anderen Aknetherapeutika beeinflusst die Substanz alle vier wesentlichen pathogenetischen Faktoren der Akne: intrafollikuläre Hyperkeratose, Androgen-abhängige Seborrhö, mikrobielle Hyperkolonisation mit Propionibacterium acnes sowie Entzündung und Immunreaktion. Unerwünschte Nebenwirkungen, die bei der oralen Einnahme auftreten können, zumeist dosisabhängig und reversibel sind, betreffen insbesondere die Haut und Schleimhaut (Cheilitis sicca, Dermatitis facialis) sowie Laborveränderungen (Erhöhung von Transaminasen und Serumlipiden), daneben erfordert die Teratogenität bei Frauen im gebärfähigen Alter eine strikte Einhaltung von Sicherheitsmaßnahmen, die eine sichere Kontrazeption und regelmä- 
ßigen Schwangerschaftsausschluss einschließen. Isotretinoin ist eine hochwirksame, mittlerweile in Europa seit über zwei Jahrzehnten in der Aknetherapie bewährte Substanz, die in ausreichender Dosierung und Behandlungsdauer nach Beendigung der Therapie oftmals zu einer bleibenden, mitunter lebenslangen Erscheinungsfreiheit führt und sich hierdurch von allen anderen Aknetherapeutika positiv abhebt [9].

Im Zusammenhang mit dem vorgestellten Fall ist von Bedeutung, dass die Substanz in der Leber über Enzyme des Cytochrom-P450-Systems metabolisiert wird und daher eine Reihe möglicher Interaktionen mit anderen Pharmaka aufweist [6]. Aufgrund der fast vollständigen Bindung an Plasmaproteine (>99\%) ist eine Interaktion zwischen Isotretinoin und Antiepileptika durch Verdrängung von den Proteinbindungsstellen zumindest theoretisch vorstellbar, jedoch sind diese Arzneimittelinteraktionen bisher nicht in klinischen Studien untersucht worden. Es gibt lediglich Hinweise verschiedener Autoren, die eine derartige Arzneimittelinteraktion nahelegen.

Marsden [8] berichtete über eine Interaktion zwischen Isotretinoin und Carbamazepin, die bei einem Aknepatienten aufgetreten war. Die Metabolisierung von Carbamazepin erfolgt in der Leber. Ein aktiver Metabolit der Substanz ist deren 10,11-Epoxit. Es wäre also denkbar, dass Isotretinoin den Stoffwechsel beider Substanzen (Carbamazepin und dessen Metaboliten) beeinflusst. Diese Vermutung wurde bei einem Aknepatienten überprüft, der wegen einer Epilepsie mit Grand-Mal-Anfällen behandelt wurde. Die Konzentrationen von Carbamazepin und dessen Metaboliten nahmen während der Therapie mit Isotretinoin ab. Es wurde angenommen, dass die Veränderungen auf eine verringerte Absorption oder verstärkte Elimination von Carbamazepin und dessen Metaboliten zurückzuführen waren.

Macdonald-Hull und Cunliffe [7] fanden bei sechs Aknepatienten keinen sicheren Hinweis auf eine Interaktion zwischen Isotretinoin und den eingesetzten Antiepileptika (Phenytoin, Natriumvalproat und Carbamazepin, einzeln oder in Kombination verabreicht). Die Epilepsie wurde medikamentös beherrscht, ohne dass Veränderungen auftraten, die mit Nebenwirkungen der verabreichten Antiepileptika sicher in einen Zusammenhang zu bringen waren. Lediglich ein Patient entwickelte in der sechsten Behandlungswoche einen epileptischen Anfall, der jedoch nach Ansicht der Autoren nicht mit der Medikation zusammenhing, sondern den progredienten Verlauf der Erkrankung bei diesem Patienten widerspiegelte.

Fernandez Vozmediano und Mitarbeiter [2] wiesen auf eine mögliche Interaktion zwischen Isotretinoin und Phenobarbital hin. Selbst in hohen Dosen erwies sich das Retinoid bei einem Patienten mit Epilepsie, der an einer durch Acne inversa komplizierten Acne conglobata litt, als wirkungslos. Die Autoren führten ihre Beobachtung auf eine Induktion von Leberenzymen durch Phenobarbital zurück, die eine beschleunigte Metabolisierung von Isotretinoin zur Folge hatten.

Von Fex und Mitarbeitern [3] stammen weitere Hinweise auf eine mögliche Interaktion zwischen Isotretinoin und Antiepileptika. Sie konnten nachweisen, dass bei Patienten, die mit Phenytoin, Carbamazepin und Valproinsäure in therapeutischen Dosen behandelt wurden, die endogenen Konzentrationen von alltrans- (Tretinoin) und 13-cis-Retinsäure (Isotretinoin) signifikant abnahmen. Die Autoren führten diese Veränderungen auf die Induktion von Retinoid-metabolisierenden Enzymen in der Leber zurück. Die Beeinflussung des Retinoidstoffwechsels wurde für die Teratogenität der Antiepileptika verantwortlich gemacht.
Die bisher vorliegenden, wenngleich nicht widerspruchsfreien Mitteilungen legen die Möglichkeit einer Interaktion zwischen Isotretinoin und Antiepileptika nahe. Daher ist aufgrund potenzieller Arzneimittelinteraktionen eine regelmäßige Kontrolle der Antiepileptikaspiegel notwendig, wie sie bei dem vorgestellten Patienten durchgeführt wurde. Auch klinische Kontrollen zum Therapieverlauf und zur Einstellung des epileptischen Anfallsleidens sind in regelmäßigen Abständen zu wiederholen. Darüber hinaus sollten der Patient und auch der behandelnde Neurologe über mögliche Arzneimittelinteraktionen informiert werden. Das bedeutet aber auch, dass Patienten mit einer schwerwiegenden Hauterkrankung wie einer Acne conglobata und einer gleichzeitig vorhandenen chronischen, dauerhaft behandlungsbedürftigen Erkrankung wie einer Epilepsie nicht von einer systemischen Isotretinointherapie ausgeschlossen werden. Denn obwohl zumindest theoretisch Arzneimittelinteraktionen möglich sind, so zeigt sich doch in der klinischen Praxis, dass durch regelmäßige Kontrollen der Medikamentenspiegel und eventuelle Dosisanpassungen des Isotretinoins Aknepatienten erfolgreich und komplikationslos behandelt werden können [1]. Die Tatsache, dass Akne als chronische Dermatose mit einer hohen psychosozialen Belastung des Patienten und schwerwiegenden, oft lebenslangen Komplikationen wie Narbenbildung einhergehen kann, sollte die Therapieentscheidung bei gegebener Indikation in Richtung einer systemischen Isotretinointherapie lenken.

\section{Fazit \\ $\nabla$}

Der vorgestellte Fall zeigt eindrücklich, dass eine systemische Isotretinointherapie von schwerer Akne auch bei gleichzeitigem Vorhandensein von Begleiterkrankungen erfolgen kann. Die Angaben aus der Literatur und auch eigene klinische Erfahrungen weisen darauf hin, dass die kombinierte Gabe von Isotretinoin und Antiepileptika bei Aknepatienten keine größeren Probleme aufwirft, auch wenn grundsätzlich Vorsicht geboten und den zumindest theoretischen Risiken von Arzneimittelinteraktionen durch klinische und serologische Verlaufskontrollen Rechnung zu tragen ist. Isotretinoin kann mit Antiepileptika kombiniert werden, wenn die Indikation zu einer Systemtherapie aufgrund der Schwere und Ausdehnung des Hautbefundes gegeben ist. Die Retinoidtherapie kann hierbei in der üblichen Dosis eingeleitet werden, ohne dass auf eine niedrigere Anfangsdosis zurückgegriffen werden müßte. Der vorgestellte Fall bestätigt, dass bei einer Epilepsie keine Dosisveränderung des Isotretinoins erforderlich ist. Selbstverständlich stellt eine enge Kooperation von Dermatologen und Neurologen die Voraussetzung für den Therapieerfolg dar.

\section{Abstract}

\section{Systemic Treatment with Isotretinoin in a Patient with Acne Conglobata and Epilepsy Controlled with Lamotrigine and Valproic Acid $\nabla$}

A 21-year-old patient with acne conglobata who received lamotrigine and valproic acid for epilepsy was treated with oral isotretinoin $40 \mathrm{mg}$ daily (according to $0.5 \mathrm{mg} / \mathrm{kg}$ body weight daily). Serum levels of antiepileptics were checked regularly. Since treatment led to significant improvement after four months, iso- 
tretinoin dosage could be reduced to $30 \mathrm{mg}$ daily. Pathological changes of serum levels of antiepileptics could not be observed during the complete treatment period. In severe acne, isotretinoin should be given to patients treated with antiepileptics if potential drug interactions are considered.

\section{Literatur}

1 Cunliffe WJ, Stables GI. Optimum use of Isotretinoin. J Cutan Med Surg 1996; $1: 2-20$

2 Fernandez Vozmediano JM, Casas Gomez VE, Alonso Blasi N, Galiana Martinez PJ, Luque Barea MA. Resistencia terapéutica al isotretinoin en un caso de acné conglobata en tratamiento anticomical. Actas Dermato-Sifil 1990; 81: 185- 187

3 Fex G, Larsson K, Andersson A, Berggren-Soderlund M. Low serum concentration of all-trans and 13-cis retinoic acids in patients treated with phenytoin, carbamazepine and valproate. Possible relation to teratogenicity. Arch Toxicol 1995; 69: 572-574

4 Greenwood R, Fenwick PB, Cunliffe WJ. Acne and anticonvulsants. Br Med J 1983; 287: 1669- 1670

5 Hesse S, Berbis P, Lafforgue P, Acquaviva PC, Pastor MJ, Privat Y. Acné et enthésiopathie au cours d'un traitement anti-épileptique. Ann Dermatol Venereol 1992; 119: 655-658

6 Larsen FG, Nielsen-Kudsk F, Jakobsen P, Weismann K, Kragballe K. Pharmacocinetics and therapeutic efficacy of retinoids in skin disease. Clin Pharmacokinet 2002; 23: $42-61$

7 Macdonald-Hull S, Cunliffe WJ. The safety of isotretinoin in patients with acne and systemic diseases. J Dermatol Treat 1989; 1: 35-37

8 Marsden JR. Effect of isotretinoin on carbamazepine pharmacokinetics. Br J Dermatol 1988; 119: $403-404$

9 Plewig G, Albrecht G, Henz BM, Meigel W, Schöpf E, Stadler R. Systemische Behandlung der Akne mit Isotretinoin: Aktueller Stand. Hautarzt 1997; 48: $881-885$

10 Plewig G, Jansen T. Isotretinoin. In: Plewig G, Wolff $\mathrm{H}$ (Hrsg). Fortschritte der praktischen Dermatologie und Venerologie, Bd 16. Berlin: Springer, 1999: $127-140$

\section{Buchbesprechung}

Erlebniswelt Naturwissenschaften.

Moleküle, Zellen, Pflanzen, Tiere, Menschen

Reitz M (Hrsg.)

Aulendorf: Editio Cantor, 2008. 359 S., mit meist farbigen Abb. Kart., $28 €$

ISBN 978-3-87193-372-1

In dem hier vorgelegten, deutschsprachigen Buch hat Manfred Reitz eine Artikelsammlung vorgelegt, für alle die wohlformulierte und verständliche Informationen aus Biowissenschaften und Medizin suchen. Und das ist auch das Metier des Forschers und Wissenschaftsjournalisten, der Biologie mit den Schwerpunkten Biochemie und Molekularbiologie aber auch Publizistik studiert hat. Die Beiträge in diesem Buch sind in einer Kolumne aus Wissenschaft und Forschung in den Jahren zwischen 1994 und 2007 in der Fachzeitschrift „die pharmazeutische Industrie pharmind“ erschienen. Aufgeteilt in 5 Groß-Kapitel finden sich 10 - 14 Unterkapitel, kurz und unterhaltend geschrieben. Im Kapitel Moleküle wird u.a. die DNA-Welt statt der alten Ursuppentheorie vorgestellt. Das Kapitel Zellen befasst sich z.B. mit Zellteilung und deren Ende, Altern und Mitochondrien, der „Festung Tumor“ und genetischen Fragestellungen, sowie mit Elektromagnetischen Feldern und Linsen. Im Kapitel Pflanzen findet sich auch die Diskussion um Methan und Treibhauseffekt, mit dem Hinweis auf die Methanbildung durch Wiederkäuer am Beispiel Rind. Aber auch das Kapitel Biofilme, Wein und Klima und wie Pflanzen sich durch Alarmstoffe selbst schützen, sind spannend zu lesen. Im Kapitel Tiere lernt man dann, was Kladogenese ist, nämlich, die Aufspaltung einer Mutterart in zwei Tochterarten, die erst dem Leben in der Evolution seine Vielfalt gab. Darüber hinaus, u. a. warum der Pottwal so einen großen Kopf hat und einiges zu den japanischen Muscheltaucherinnen. Das letzte Kapitel zum Menschen ist ein Kaleidoskop mit z.B. einem Kapitel zum aufrechten Gang, zu Knochenfunden, zu DNA und Wanderwegen, Leben in Kälte und Hitze und die Hundertjährigen, neben vielen anderen Themen. Schemazeichnungen und Farbabbildungen ergänzen die einzelnen Kapitel und jeweils 3 Literaturangaben.

Das vorliegende, gut lesbare Buch trägt seinen Titel einer Erlebniswelt zu Recht. Auch die vom Autor selbst gewählte Charakterisierung als Anthologia passt, denn in seiner ursprünglichen Bedeutung bezeichnet der Begriff eine Sammlung von „Blumen und Blüten“ - will heißen, es wurde Schönes zusammengetragen.

Christiane Bayerl, Wiesbaden 